\title{
Understanding the complex anatomy of the middle turbinate via educational origami*
}

\section{Reda Kamel}

Department of Otorhinolaryngology, Cairo University Hospital, Cairo, Egypt
Rhinology Online, Vol 1: 154 - 159, 2018

http://doi.org/10.4193/RHINOL/18.072

*Received for publication:

September 5, 2018

Accepted: October 13, 2018

Published: October 16, 2018

\begin{abstract}
Background: The middle turbinate is an important landmark during endoscopic sinus procedures. Well orientation of the appropriate planes, attachments and relations of the different parts of the middle turbinate helps achieve safe and effective endoscopic sinus surgery.
\end{abstract}

Aim of work: To discuss the complex anatomy of the middle turbinate via a paper model of the middle turbinate and to validate its impact on clarifying the planes and attachments of the different parts of the middle turbinate.

Study design: descriptive.

Methods: Utilizing origami, a paper model of the middle turbinate was introduced by the author resembling its three parts. The model was used to demonstrate the planes and attachments of the different parts of the middle turbinate. Ten MCQs were administered to 76 candidates, prior and post demonstration, during three basic endoscopic sinus surgery courses. The pre and post scores were statistically analyzed.

Results: The mean of pre-total score was 6.05 (range 0-10, SD 2.39) and the mean of the post-total score was 9.22 (range 7-10, SD 1.05). The difference of pre and post total score means was 3.17 (SD 2.32) which showed high statistical significance.

Conclusion: The Kamel's middle turbinate paper model is a valid educational tool. It is simple to assemble and useful to interpret the planes, attachments and relations of the different parts of MT. Moreover it has many fruitful clinical applications that may help achieve effective and safe endoscopic sinus surgery.

Key words: ethmoid sinus, nasal cavity, paranasal sinuses, sinusitis, turbinates

\section{Introduction}

Endoscopic sinus surgery (ESS) has become the state of the art in management of most cases of persistent chronic rhinosinusitis, sinonasal polyps and fungal rhinosinusitis ${ }^{(1-3)}$. Proper understanding of the endoscopic anatomy of the sinonasal region is mandatory to avoid complications ${ }^{(1,4-7)}$.

The middle turbinate (MT) is a vital anatomical landmark that guides the surgeon to perform safe and effective $\operatorname{ESS}(5,6,8,9)$. It is composed mainly of three parts, that are oriented in different planes and have definite attachments to the lateral nasal wall, orbit and skull base $\mathrm{e}^{(5,6,9)}$.
During basic ESS courses in a lot of countries within the last twenty years, the author noticed that many participants, especially young doctors, failed to understand and grasp easily the orientation and attachments of the different parts of MT. This is despite excellent lectures and/or demonstrations. This aroused the need for a simple model for the presentation of the MT and understanding its complex anatomy.

The aim of work is to demonstrate an original paper model of the MT that helps understand the planes, attachments and relations of the different parts of the MT and to validate its efficacy. 


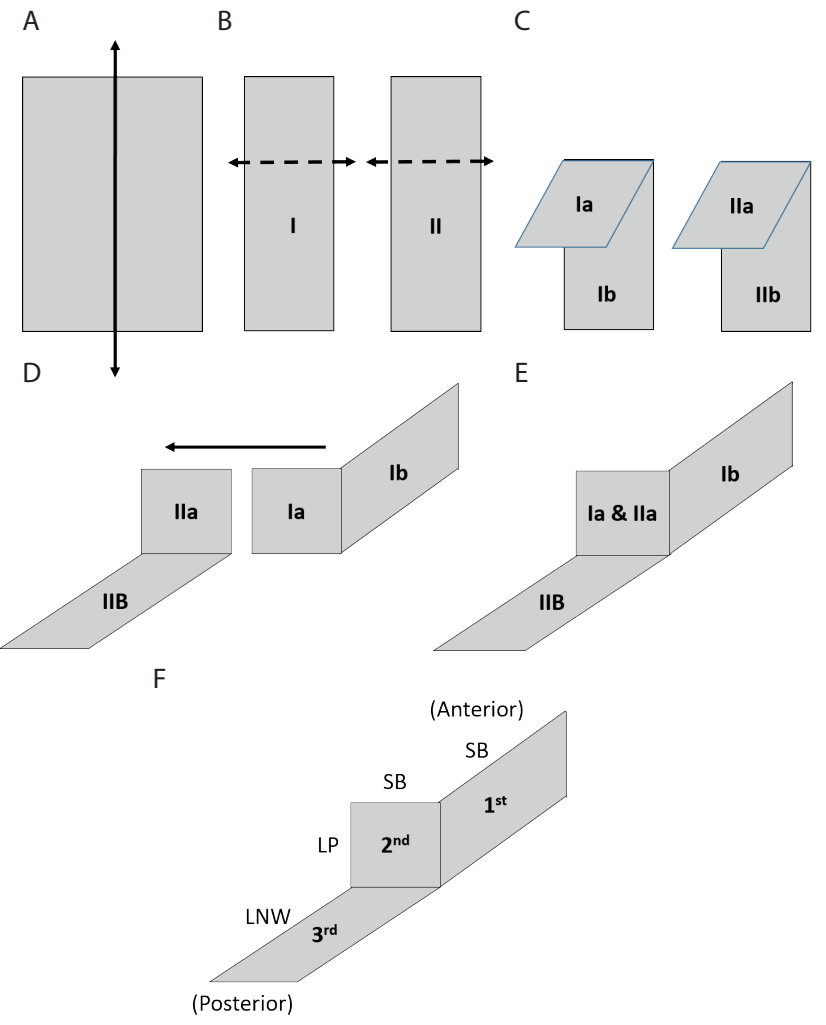

Figure 1. A, B) A sheet of paper (A4) is cut longitudinally into two equal parts, namely I and II. C) Each piece is bent perpendicularly into two unequal parts, one-third and two-thirds, namely short la \& long lb and short lla \& long llb respectively. D,E) The short parts of both pieces of paper namely la and lla are applied on each other, in a coronal plane whereas the long part of paper I (Ib) is rendered in a sagittal plane and the long part of paper II (IIb) is rendered in a transverse plane. This resembles the left MT. F) The MT is composed of three parts; first, second and third parts. The first anterior sagittal oriented first part (Ib) is perpendicular and attached to skull base (SB) superiorly. The third posterior transversely oriented part (IIb) is perpendicular and adherent to lateral nasal wall laterally (LNW). The basal lamella is the second part of the MT and is coronal oriented (la \& Ila) and perpendicular to both the skull base superiorly and the lamina papyracea laterally.

\section{Materials and Methods}

A paper model of the MT was introduced by the author to resemble its three parts via origami. The model was utilized to demonstrate the planes and attachments of the MT. Before and after the demonstration, 10 MCQs (Appendix 1) were administered to 76 candidates during three basic ESS courses held in Egypt (21, 31 and 24 delegates respectively). Questions 1-3 aim the orientation and planes of the three parts of the MT, Questions 4-5 target the location and shape of basal lamella (BL) and questions 6-10 address the different attachments of the BL. Correct answer was scored one and the incorrect zero. Each candidate supplied his/her age, degree, position, province, years of experience of ENT and ESS and number of previous ESS

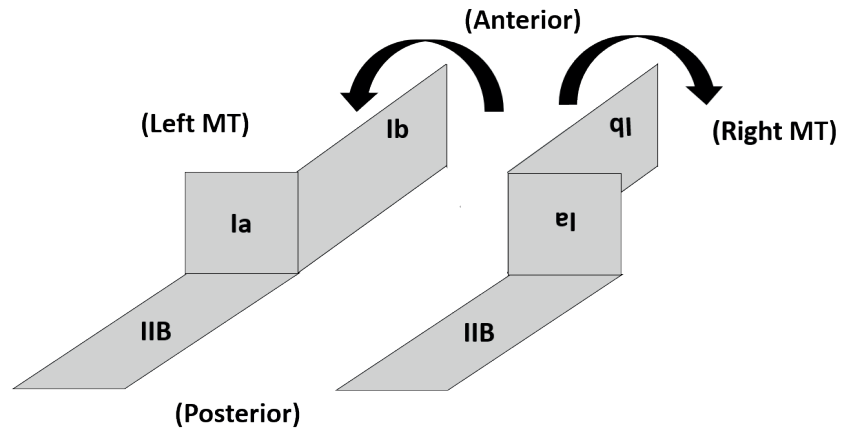

Figure 2. The paper model, as seen from behind, is changed from left to right design and vice versa by rotating only the first piece of paper (la \& Ib) $180^{\circ}$ anticlockwise and clockwise, respectively.

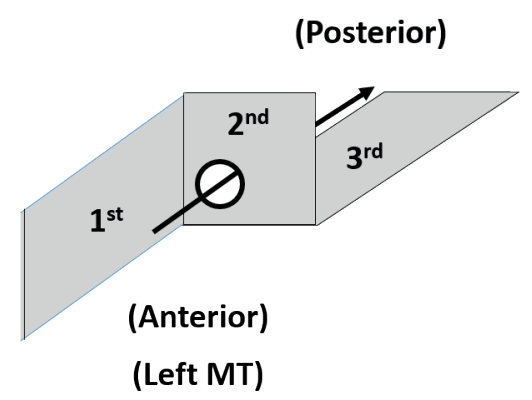

Figure 3. The left basal lamella, visualized from front, is opened inferior and medial which is the safest location. This is away from the superior skull base and lateral lamina papyracea.

courses attended. Candidates with missing personal data and/or incomplete answers were excluded.

The paper model of the three parts of the MT:

A sheet of paper (A4) is cut longitudinally into two equal parts, namely I and II (Figure 1A). Each piece of paper is then bent perpendicularly into two unequal parts, one-third and two-thirds namely short la \& long Ib and short Ila \& long Ilb, respectively (Figure 1B \& C). The short parts of both pieces of paper namely la and Ila are applied on each other, in a coronal plane whereas the long part of paper I ( $\mathrm{lb}$ ) is rendered in a sagittal plane and the long part of paper II (Ilb) is rendered in a transverse plane (Figure 1D \& E).

The sagittal oriented part (lb) simulates the first anterior part of the left MT, the transversally oriented part (IIb) resembles the third posterior part of MT, and the middle duplicated coronal oriented part (la \& Ila) resembles the BL or second middle part of MT (Figure 1F). Then the model is used to demonstrate the following:

1- MT orientation and attachments:

The MT is composed of three parts; first, second and third parts. 
The first anterior sagittal oriented part (lb) is parallel to lamina papyracea laterally and the nasal septum medially and perpendicular and attached to the skull base superiorly. The third posterior transversely oriented part (IIb) is parallel to nasal floor inferiorly and skull base superiorly and perpendicular and adherent to lateral nasal wall laterally. There should be a changing part between the two differently oriented first and third parts of the MT. It is the second middle part which is the BL of the MT. It is coronal oriented (la \& Ila) and perpendicular to both the skull base superiorly and the lamina papyracea laterally. Accordingly, the $\mathrm{BL}$ is generally rectangular in shape and has four borders and attachments. It is attached superiorly to the skull base, laterally to the lamina papyracea, medially to the first part of the MT and inferiorly to the third part of the MT (Figure 1F).

\section{2- Right and left layout of MT:}

The first piece of paper only, namely short la \& long lb, is then rotated anticlockwise $180^{\circ}$ to change from the left to the right layout of the MT (Figure 2).

3- Location of bulla ethmoidalis and posterior ethmoid air cells:

Then one should be able to imagine the location of the bulla ethmoidalis anterior to the $\mathrm{BL}$ and the location of the posterior ethmoid behind the BL.

\section{4- BL opening:}

The model is then used to show where to penetrate the BL. The $\mathrm{BL}$ is penetrated inferior and medial which is the safest site. A pen is used to follow the lateral aspect of the first sagittal part of MT from anterior to posterior and one should appreciate the change from the sagittal plane to coronal plane of BL. Then the pen is used to follow the inferior aspect of the third transverse part of MT from posterior to anterior and one should appreciate the change from the transverse to the coronal plane of $\mathrm{BL}$. The meeting point is the safest and optimum site of opening of the $B L$ which is then done by the tip of the pen. Then the hole is widened superior and lateral towards skull base and lamina papyracea respectively (Figure 3). Opening of BL superior and/or lateral may induce skull base and/or orbit complications.

The same 10 MCQs were re-administered to the candidates. Then the correct answers were displayed and the delegates were asked to make their own models.

\section{Statistical analysis}

Data were statistically described in terms of mean \pm standard deviation ( $\pm S D$ ), median and range, or frequencies (number of cases) and percentages when appropriate. Comparison of numerical variables between the study groups was done using Student $t$ test for independent samples in comparing 2 groups of normally distributed data and Mann Whitney $U$ test for independent samples for comparing not-normal data. Comparison of normally distributed numerical variables between more than two groups was done using Kruskal Wallis test. Comparison
Table 1. The range, mean and standard deviation of pre and post scores of each question separately and total scores.

\begin{tabular}{|lllll|}
\hline Question & Minimum & Maximum & Mean & SD \\
\hline Pre-Q1 & 0 & 1 & 0.64 & 0.482 \\
\hline Pre-Q2 & 0 & 1 & 0.53 & 0.503 \\
\hline Pre-Q3 & 0 & 1 & 0.49 & 0.503 \\
\hline Pre-Q4 & 0 & 1 & 0.71 & 0.457 \\
\hline Pre-Q5 & 0 & 1 & 0.62 & 0.489 \\
\hline Pre-Q6 & 0 & 1 & 0.70 & 0.462 \\
\hline Pre-Q7 & 0 & 1 & 0.68 & 0.468 \\
\hline Pre-Q8 & 0 & 1 & 0.74 & 0.443 \\
\hline Pre-Q9 & 0 & 1 & 0.53 & 0.503 \\
\hline Pre-Q10 & 0 & 1 & 0.42 & 0.497 \\
\hline Pre-Total & 0 & 10 & 6.05 & 2.399 \\
\hline Post-Q1 & 0 & 1 & 0.99 & 0.115 \\
\hline Post-Q2 & 0 & 1 & 0.95 & 0.225 \\
\hline Post-Q3 & 0 & 1 & 0.22 & 1.053 \\
\hline Post-Q4 & 1 & 1 & 0.96 & 0.196 \\
\hline Post-Q5 & 0 & 1 & 1.00 & 0.000 \\
\hline Post-Q6 & 0 & 1 & 0.95 & 0.225 \\
\hline Post-Q7 & 0 & 1 & 0.196 \\
\hline Post-Q8 & 0 & 1 & 0.196 \\
\hline Post-Q9 & 0 & 1 & 0.325 \\
\hline Post-Q10 & 0 & 1 & 0.367 \\
\hline Post-Total & $\mathbf{1}$ & 1 & 0.443 \\
\hline
\end{tabular}

$\mathrm{SD}=$ standard deviation

between pre and post-data was done using paired t test in normally distributed data and Wilcoxon signed rank test for paired (matched) samples when data were not normally distributed. Correlation between various variables was done using Spearman rank correlation equation. $p$ values less than 0.05 was considered statistically significant. All statistical calculations were done using computer program IBM SPSS (Statistical Package for the Social Science; IBM Corp, Armonk, NY, USA) release 22 for Microsoft Windows.

\section{Results}

Table 1 shows the range, mean and standard deviation of pre and post scores of each question separately and total scores. The difference of pre and post total score means was 3.17 (SD 2.32) which showed high statistical significance ( $p$ value 0.000 ). All questions, Q1-10, displayed significant statistical difference regarding pre and post scores (Figure 4).

After introduction to the model Q3 (posterior MT) and Q2 (middle part of MT) revealed maximum benefit (50\% \& 43.4\%, respectively). Q10 \& Q9 (attachments of MT) exhibited the least 


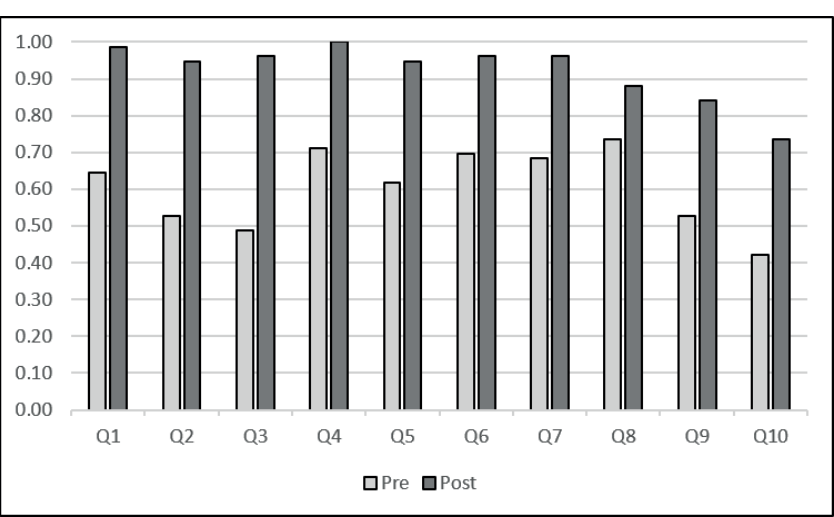

Figure 4. Pre and post scores before and after demonstration of the Kamel's paper model of middle turbinate.

improvement (26.3\% \& 15.8\%, respectively).

Q8 (lateral attachment) showed highest awareness prior to the initial assessment (mean 0.74). Q10 presented least awareness before model presentation (mean 0.42). Q4 (BL of MT) regarding the $\mathrm{BL}$ being the second part of MT exhibited maximum awareness after model demonstration (mean 1). Q10 and Q9 displayed least awareness after model demo concerning the medial and inferior attachments of BL to the first sagittal and third transverse parts of MT respectively (mean $0.74 \& 0.84$ ).

There was no statistical difference regarding the age of participants (range 25-60 years, mean 34.6), venue of the three courses, scientific degree acquired by the participants, work area whether urban or suburban, number of previous ESS courses attended and/or number of years of ENT experience. There was significant statistical difference regarding the position of the participants ( $p$ value 0.038 ) where the residents and specialists showed highest pre and post score difference followed by the consultant and lastly the assistant lecturers. There was high statistical difference regarding the number of years of ESS experience (range $0-20$ years, mean 2.18 , std. deviation $0.008, p$ value 0.008 ).

\section{Discussion}

The MT is an important landmark in ESS ${ }^{(5,6,8,9)}$. The most anterior and superior insertion of the MT is adjacent to the crista ethmoidalis of the maxilla. The posterior end is attached to the crista ethmoidalis of the perpendicular process of the palatine bone. The area between comprises three parts. The anterior third of the MT inserts vertically into the skull base at the lateral edge of the lamina cribrosa. The middle third turns laterally across the skull base to the lamina papyracea, where it turns inferiorly. The most posterior segment becomes horizontal to form the roof of the posterior third of the middle meatus. The insertion of the MT thus lies in three different planes, sagittal, frontal and horizon$\mathrm{tal}^{(5,6,9)}$.

A lot of tools have been presented to illustrate the MT including printed diagrams, plastic models, computer animation and $3 \mathrm{D}$ reconstruction, tri-planar and 3D CT reconstruction, virtual endoscopy, and dextroscope virtual reality, fresh cadavers and/ or dry skulls $5,10,11)$. Unfortunately, many of these models do not reach final conclusion of MT anatomy regarding planes, attachments and/or relations of the different parts. Printed diagrams are helpful but two dimensions ${ }^{(6,9)}$. Computer animation and $3 \mathrm{D}$ reconstruction are informative but in most of the cases fail to achieve full awareness of the middle turbinate especially by young doctors. Tri-planar and 3D CT reconstruction are very beneficial but sometimes difficult to interpret by inexperienced surgeons ${ }^{(12,13)}$. The plastic models are useful but usually small in size and difficult to demonstrate all details of planes, attachments and relations of the $\mathrm{MT}^{(14)}$. Virtual endoscopy and dextroscope virtual reality are promising but expensive and looking for further developments ${ }^{(10,11)}$. Dry skulls are good especially when sagittally cut but unfortunately it is usually hard to obtain in many centers. Moreover, the bony skeleton of the middle turbinate is relatively friable and missing in many dry skulls ${ }^{(15)}$. Fresh cadavers are exceptional, they offer true dimensions and configuration of the MT but they are expensive and not always available ${ }^{(5)}$.

The middle turbinate exhibiting three different plans, coronal, axial and sagittal and being attached to many nearby important structures including the skull base and orbit usually remains a big challenge to inexperienced endoscopic surgeons. Losing orientation during endoscopic surgery may lead to missed pathology and/or insult to skull base and /or orbit ${ }^{(5)}$. Origami is the Japanese art of folding paper into decorative shapes and figures. The author utilized the art of paper folding in making a MT model. 10 MCQ were designed to validate the usefulness of the MT paper model.

Question 1-3 aimed the orientation of the three parts of the MT. Before model presentation, the awareness of the sagittal orientation of the first anterior part, the coronal orientation of the second middle part and the axial orientation of the posterior third part were $64.5 \%, 52.6$ and $48.7 \%$ respectively. These awareness increased to $98.7 \%, 94.7$ and $96.1 \%$ respectively after model presentation. The differences were statistically significant ( $p$ value $0.000,0.000$ and 0.000 ).

The $\mathrm{BL}$ is the second part of the $\mathrm{MT}^{(5,6,8,9)}$. Question 4 aiming this point was answered correctly by $71.1 \%$ of the participants before model demonstration and by $100 \%$ after. The difference was statistically significant ( $p$ value 0.000 )

The $\mathrm{BL}$ is rectangular in shape ${ }^{(8)}$. Question 5 which targeted this point was already known by $61.8 \%$ of participants before the model being presented and by $94.7 \%$ after. The difference was statistically significant ( $p$ value 0.000 ).

Most literature usually stress upon the fact that the BL is attached to the lamina papyracea, a fact concentrated upon to avoid insult to the orbit during $\operatorname{ESS}^{(5,6,8,9)}$. Unfortunately, this usually 
gives the false impression that the $B L$ has only one attachment. Question 6 addressed the fact that the BL has four different attachments and was correctly answered by $69.7 \%$ before model presentation and increased to $96.1 \%$ after. The difference was statistically significant ( $p$ value 0.000 ).

Few references highlight that the BL is attached superiorly to the skull base ${ }^{(6,8)}$. And unfortunately most anatomical and endoscopic references do not emphasize the medial and inferior attachments of the $\mathrm{BL}$, being to the first and third parts of the MT respectively. This was emphasized before model presentations (question 7-10) where the awareness of the attachment of the BL laterally to the lamina papyracea was highest, superiorly to the skull base was less and inferiorly to the third part and medially to the first part of MT were least $(73.7 \%, 68.4 \%, 52.6$ and $42.1 \%$ respectively). These increased after model presentation to $88.2 \%, 96.1,84.2 \%$ and 73.7 respectively. The differences were statistically significant ( $p$ value $0.035,0.000,0.000$ and 0.000 ). It was noticed that there were still $15.8 \%$ and $26.3 \%$ unaware of the inferior and medial attachments of the BL after model presentation. This arouses the difficulty of these two attachemnts and the importance of more stress on these points during anatomical lectures and model presentation.

In general all questions (Q1-10) disclosed significant statistical difference regarding pre and post scores separately. And the difference of pre and post total score means was 3.17 (SD 2.32) which showed high statistical significance ( $p$ value 0.000 ). This emphasizes the efficacy of the MT paper model in increasing the awareness of the orientation, planes of the different parts of the $M T$, location and shape of BL and the four attachments of the BL. Moreover the current novel paper model of the MT offers some more clinical applications. The BL separates the bulla ethmoidalis anteriorly and the posterior ethmoid air cells posteriorly ${ }^{(5,6,8,9)}$. This is smartly demonstrated by the MT model.

The BL is the corridor through which one can proceed from the anterior ethmoids to the posterior ethmoids. This should begin by opening of the BL inferior and medial and never superior and/or lateral ${ }^{(5.6 .9)}$. The paper model is used to demonstrate this step simply. The insult to the orbit and/or skull base are amongst the possible most serious major complications during $\operatorname{ESS}^{(4,5,7,9)}$.
The model is utilized to demonstrate how to perform safe surgery. This is achieved by clarifying the possibility of the insult to the skull base and/or orbit in case of superior and/or lateral opening of the BL. Furthermore, floppy MT may lead to synechia formation and the recurrence of sinusitis ${ }^{(16)}$. The importance of the integrity of the first sagittal and third transverse parts of MT are well shown by the model to avoid floppy MT.

The new Kamel's MT model is easy to assemble by both the instructors and trainees. There is no need for any special materials and/or tools, just a sheet of A4 paper. It is practical, cheap and reproducible. It is simple and an onsite demo, anytime and anywhere. It can easily be interchanged between right and left layout. It is exceptional in case of beginners. In fact it is an unconventional method of MT demonstration being an interactive part during courses and/or lectures.

Nonetheless, the Kamal's paper model needs training to master. It does not reflect the true dimensions, exact shape and/or precise orientation of the MT. It should be considered as a supplementary tool and does not replace other illustrative tools, cadaver dissection and/or intraoperative integration.

It is worthy to mention that some anatomical variations and/ or pathological abnormalities may obviate making full use of the MT as a landmark ${ }^{(6,17)}$. Moreover, in recurrent cases, the MT may be mutilated, amputated, cut or adherent to lateral nasal wall. This may preclude utilizing the MT as a navigator in many revision surgeries.

\section{Conclusion}

The Kamel's MT paper model is a valid educational tool. It is simple to assemble and useful to interpret the planes, attachments and relations of different parts of MT. Moreover it has many fruitful clinical applications to achieve safe and effective ESS.

\section{Authorship contribution}

$\mathrm{KR}$ is responsible for all aspects of the manuscript.

\section{Conflict of interest}

None

\section{References}

1. Fokkens WJ, Lund VJ, Mullol J, Bachert C, Alobid I, Baroody F, et al. et al., European position paper on rhinosinusitis and nasal polyps. Rhinol Suppl. 2012; 23: 1-229.

2. Rudmik L, Mace J, Soler ZM, Smith TL. Long-term utility outcomes in patients undergoing endoscopic sinus surgery. Laryngoscope 2014; 124(1): 19-23.

3. Noon E, Hopkins C. Review article: outcomes in endoscopic sinus surgery. BMC Ear, Nose and Throat Disorders, sBMC series - open, inclusive and trusted 2016; 16:9.

4. Stankiewicz JA. Complications of endoscop- ic sinus surgery. Otolaryngologic Clinics of North America 1989; 22(4): 749-758.

5. Stammberger $H$, Hawke M. Essentials of functional endoscopic sinus surgery. Pb. Elsevier Health Sciences, Mosby, 1993. p. 18-19.

6. Stammberger HR, Kennedy DW. Paranasal sinuses: anatomic terminology and nomenclature. Ann Otol Rhinol Laryngol Suppl. 1995; 167: 7-16.

7. Hopkins C, Browne JP, Slack R, Lund VJ, Topham J, Reeves BC, et al., Complications of surgery for nasal polyposis and chronic rhinosinusitis: the results of a national audit in England and Wales. Laryngoscope 2006; 116(8): 1494-1499.

8. Kamel R. Endoscopic anatomy of the lateral nasal wall, ostiomeatal complex and anterior skull base: A step-by-step Guide. EndoPress, Tuttlingen, Germany, 2000; 1-34.

9. Lund VJ, Stammberger HR, Fokkens WJ, Beale T, Bernal-Sprekelsen M, Eloy P, et al., European position paper on the anatomical terminology of the internal nose and paranasal sinuses. Rhinol Suppl. 2014; 24: 1-34.

10. Gilani S, Norbash AM, Ringl H, Rubin GD, Napel S, Terris DJ. Virtual endoscopy of the paranasal sinuses using perspective volume 
rendered helical sinus computed tomography. Laryngoscope 1997; 107 (1): 25-29.

11. Tolsdorff $B$, Pommert A, Hohne KH, Petersik A, Pflesser B, Tiede U, Leuwer R. Virtual reality: a new paranasal sinus surgery simulator. Laryngoscope 2010; 120 (2): 420-426.

12. Gotwald TF, Zinreich SJ, Corl F, Fishman EK. Three-Dimensional Volumetric Display of the Nasal, Ostiomeatal Channels and Paranasal Sinuses, AJR 2001; 176: 241-245.

13. Wormald PJ, Endoscopic Sinus Surgery: Anatomy, Three-Dimensiona Reconstruction and surgical Technique. Third edition, New York, Stuttgart: Thieme, 2013, p. 107-110.

14. Briner HR, Simmen D, Jones N, Manestar D, Manestar M, Lang A, Groscurth P. Evaluation of an anatomic model of the paranasa sinuses for endonasal surgical training. Rhinology. 2007; 45(1):20-23.

15. Bradoo R, Anatomical Principles of Endoscopic Sinus Surgery: A step by step approach. New Delhi: JAYPEE Brothers Medical publishers (P) LTD, 2005, p. 19-22.

16. Lee HY, Kim CH, Kim JY, Kim JK, Song MH, Yang $\mathrm{HJ}$, et al., Surgical anatomy of the middle turbinate. Clin Anat. 2006; 19 (6): 493496.

17. Hosemann WG, Weber R, Keerl R, et al., Minimally Invasive Endonasal Sinus Surgery: Principles, Techniques, Results, Complications, Revision Surgery. 2000. p. $7-8$
Reda Kamel

Professor of Rhinology

Cairo University

87 Manial Street

Manial

Cairo - 11451

Egypt

Mobile: +201222137172

E-mail: rhinology@redakamel.com website: www.redakamel.com

Appendix 1. The 10 MCQs:

Q1: The anterior part of middle turbinate [MT] is: A-Sagittal oriented

B- Coronal oriented

C- Axial oriented

D- None of the above

Q2: The middle part of MT is:

A-Sagittal oriented

B- Coronal oriented

C-Axial oriented

D- None of the above

Q3: The Posterior part of MT is:

A-Sagittal oriented

B- Coronal oriented

C- Axial oriented

D- None of the above

Q4: The basal lamella of MT is the:

A- First part of MT

B- Second part of MT

C-Third part of MT

D- None of the above

Q5: The basal Lamella of MT is generally:

A-Triangular in shape

B- Rectangular in shape

C- Round in shape

D- None of the above
Q6: The basal lamella of MT is attached to the:
A- Lamina papyracea
B- Skull base
C- First part of MT
D- Third part of MT
E- All of the above

Q7: The superior attachment of basal lamella of MT is to the:

A- Lamina papyracea

B- Skull base

C- First part of MT

D- Third part of MT

E- All of the above

Q8: The lateral attachment of basal lamella of MT is to the:
A- Lamina papyracea
B- Skull base
C- First part of MT
D- Third part of MT
E-All of the above

Q9: The inferior attachment of basal lamella of MT is to the:

A-Lamina papyracea

B- Skull base

C- First part of MT

D- Third part of MT

E- All of the above

Q10: The medial attachment of Basal Lamella of MT is to the:

A-Lamina papyracea

B- Skull base

C- First part of MT

D- Third part of MT

E- All of the above 